\section{ONOMÁVAEIN}

Revista semestral de lingüística, filología y traducción
PONTIFICIA UNIVERSIDAD

\title{
Marcadores del discurso y contextos funcionales: la reformulación como construcción discursiva
}

Discourse markers and functional contexts: reformulation as a discursive construction

\section{Fernando Polanco Martínez}

Universidad de Barcelona

España

\section{(c) $(i)$}

Fernando Polanco Martínez: Departamento de Filología Hispánica, Universidad de Barcelona, España. | Correo electrónico: polanco@ub.edu 


\section{Resumen}

En este trabajo queremos presentar la potencialidad explicativa que puede tener un tratamiento construccional de los contextos funcionales en la descripción semántico-funcional de los marcadores conversacionales. Para ello, centraremos la exposición en la función de reformulación y en algunos de los marcadores asociados de manera prototípica a dicha función, con especial énfasis en marcadores coloquiales como o sea, vamos o vaya. Con esta propuesta, creemos que pueden explicarse mejor los efectos de sentido que presentan ciertos marcadores de (re)formulación en función del contexto en que aparecen. Desde una perspectiva construccional, estas interpretaciones contextuales pueden entenderse como sentidos construccionales (Polanco, 2013a, 2014) derivados de la interacción del sentido general de la construcción discursiva que actúa de contexto funcional y el sentido prototípico del marcador.

Palabras clave: partículas discursivas; marcadores del discurso; pragmática; construcciones discursivas; gramática de construcciones en interacción.

\section{Abstract}

This work deals with the explanatory potential of applying a constructional approach of functional contexts to the semantic-functional description of conversational markers. We focus our attention in the function of reformulation and in some of its typical discursive markers in spoken Spanish, as o sea, vamos and vaya. We think that this proposal can explain better the sense effects that some refor- mulative markers present depending on the context they appear. From a constructional perspective, this contextual interpretations can be understood as constructional senses (Polanco, 2013a, 2014) derived from the interaction of the general meaning of the discursive construction, which acts as a functional context, and the prototypical sense of the discursive marker.

Keywords: discursive particles; discursive markers; pragmatics; discursive constructions; interactional construction grammar. 


\section{Introducción}

Reformular y rearticular, en tanto que funciones discursivas, forman parte de una estrategia más amplia, la formulación, con la que se reorganiza la información y tratan de resolverse los potenciales problemas comunicativos que pueden $-\mathrm{y}$ suelen- surgir a medida que se avanza en la construcción del discurso en cualquiera de sus variantes. Ahora bien, constituyen dos maneras diferentes de continuar un discurso.

Reformular supone un proceso de reinterpretación discursiva mediante el que el emisor retoma algún elemento previo de su propio discurso o del discurso de su interlocutor, explícito o implícito, para presentarlo de nuevo con una formulación que se ajusta de manera más adecuada a las expectativas discursivas creadas por la primera formulación y, por tanto, a la intención comunicativa del hablante. Es, pues, una operación de autorreflexión lingüística (Calsamiglia y Tusón, 1999: 310), un reajuste entre lo que se ha dicho y lo que se quiere decir. Con la reformulación, pues, se produce una versión nueva de algo anterior que se presenta como una opción más satisfactoria.

En este trabajo, por tanto, aplicamos una visión restringida de la reformulación. Reformular implica regresar al asunto discursivo previo para aclararlo, matizarlo, sintetizarlo o corregirlo y, en suma, expresar un contenido formulado previamente de una forma más comprensible, o más expresiva, con un (hipotético) menor es- fuerzo interpretativo. En este sentido, abarca las operaciones discursivas asociadas con la reformulación parafrástica (Gülich y Kotschi, 1983, 1995; Garcés, 2005, 2006), más la recapitulación. Sin embargo, no consideramos como una operación reformuladora funciones como la de distanciamiento ${ }^{1}$ (Rossari, 1994; Gülich y Kotschi, 1995; Portolés, 2001 [1998]; Garcés, 2005, 2006, 2008, entre otros), que será tratada como una operación de rearticulación discursiva.

La función rearticuladora², por su parte, igual que la reformuladora, es una estrategia formulativa orientada a solventar los problemas que van surgiendo en todo discurso no planificado o semiplanificado. Sin embargo, a diferencia de la reformulación, con la rearticulación no se regresa al asunto discursivo previo para aclararlo, matizarlo y volverlo a expresar de una forma más ajustada. Con esta operación se restablece la fluidez del discurso, o bien retomando el hilo del discurso tras una digresión, o bien introduciendo un nuevo tópico (ejemplo 1); bien relanzando el habla tras una pausa o una vacilación momentánea (ejemplo 2), o "rellenando" posibles silencios que podrían constituir lugares de transición pertinente (LTP) para la toma de turno por otro interlocutor (ejemplo 3), señalando de esta manera la intención del hablante de continuar con su discurso, de mantener el turno de palabra.

(1) y el de la autoescuela le dice pero tío pero ¿ide

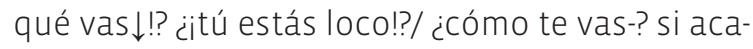

1 Los marcadores de distanciamiento actúan como mecanismos de formulación, de reorganización discursiva, a través de los cuales se presenta una desviación del punto de vista previo y se inicia una vía discursiva diferente de la anterior. Es cierto que estos marcadores también apuntan hacia lo dicho, en el sentido de que la nueva formulación anula la pertinencia de lo anterior y se presenta como la información que condicionará la continuación del discurso. Pero esta característica es, en cierto modo, común a muchos otros marcadores (si no a todos), en tanto que las operaciones semántico-pragmáticas que señalan los MD no afectan solo al segmento que introducen o sobre el que inciden, sino también a la interpretación del primer miembro y, de hecho, a la interpretación global del segmento resultante. Es decir, la interpretación del segmento introducido por un conector (pero, sin embargo, por tanto, etc.) o un operador (bueno, de hecho, en realidad, por supuesto, sin duda, por ejemplo, etc.) implica también la consideración del segmento previo para poder determinar la coherencia global de la enunciación.

2 Tomamos el concepto de "función rearticuladora" de Cortés y Camacho (2005: 211-217). Con este valor, ciertos marcadores se utilizan para solventar los problemas de formulación esperables en toda construcción discursiva no planificada, en la que abundan las interrupciones y los reajustes en la estructuración informativa (es decir, en la que aparecen mecanismos con función reparadora). 
bas de empeZAAR $\downarrow$ no has venío ningún día a CLA$\mathrm{SE} /$ vamos o s(e)a// yo si quieres yo te apunto// tú eres el que vas a tener QUE PAGAR/(..) (Briz y grupo Val.Es.Co 2002: 113 [L.15.A.2]).

(2) yo no he ido- no he sido muy de barrio (chasquido)// pero vamos recuerdo las Fallas sobre todo las Fallas// es cuando más yo/ vamos cuando yo he trabaja(d)o más en eel- juy! Perdón hee colabora(d)o o he esta(d)o así más en el barrio (...). (Preseval, [conv. 1, líneas 74-78]; http://www. uv.es/preseval/ppal.htm).

(3) Bueno, es decir, oye〈PAUSE〉 qué quieres, no sé. Dicen de que<PAUSE〉 dicen Cruz y Raya de que yo hablo inseguro. Es que pienso que<PAUSE〉 vamos, o sea, no estoy de acuerdo, pero〈PAUSE〉 vamos, no sé, ¿no? O sea, yo creo<PAUSE〉 yo que usted<PAUSE〉 vamos, no sé (...). (CREA; ¿Pero esto qué es?, 10/09/90, TVE1).

En este trabajo queremos presentar la potencialidad explicativa que puede tener un tratamiento construccional de los contextos funcionales en la descripción semántico-funcional de elementos discursivos complejos como los marcadores conversacionales. Para ello, centraremos la exposición en la función de reformulación y en algunos de los marcadores asociados de manera prototípica a dicha función ${ }^{3}$, con especial énfasis en marcadores coloquiales como o sea, vamos o vaya ${ }^{4}$. Con esta propuesta, creemos que pueden explicarse mejor los efectos de sentido que presentan ciertos marcadores de (re)formulación en función del contexto en que aparecen. Desde una perspectiva construccional, estas interpretaciones contextuales pueden entenderse como sentidos construccionales (Polanco, 2013a) derivados de la interacción del sentido general de la construcción discursiva que actúa de contexto funcional y el sentido prototípico del marcador.

\section{La reformulación como estrategia dis- cursiva}

La reformulación (parafrástica) se ha definido como una relación de equivalencia, basada o bien en una relación de equivalencia semántica gradual entre dos segmentos A y A', o bien en una predicación de identidad vehiculada por marcadores cuando esta relación de equivalencia es débil o no es interpretable a partir de los significados proposicionales (Gülich y Kotschi, 1995; Rossari, 1994). En el primer caso, la reformulación se fundamenta en el parentesco semántico que existe entre los segmentos relacionados y puede analizarse a partir de los elementos significativos comunes entre ambos enunciados. En el segundo caso, la relación de identidad se establece mediante la indicación de un marcador que presenta el enunciado que se introduce como equivalente del anterior (independientemente de que tal equivalencia se fundamente en rasgos semánticos compartidos o se recupere inferencialmente). La presencia de un marcador que establezca la relación de equivalencia dependerá del grado de equivalencia semántica que exista entre la expresión de referencia y la de reformulación: cuanto mayor sea la semejanza semántica entre ambas expresiones, menor será la necesidad de señalar una relación de equivalencia; por el contrario, cuanto mayor sea el peso inferencial en el establecimiento de la equivalencia, mayor será la necesidad de explicitar dicha relación.

En cualquier caso, el concepto de paráfrasis no debe entenderse como una relación o una predicación estática de equivalencia por la que

3 Aunque en este trabajo nos centraremos, por cuestiones de espacio, en el esquema discursivo de reformulación, esta concepción construccional puede hacerse extensible a los contextos funcionales de rearticulación, que trataremos en otro trabajo.

4 Para mayor detalle sobre la función reformuladora de vamos, ver Fuentes (1998, 2009), Santos (2003), Briz, Pons y Portolés (2008). Para vaya, ver Santos (2003), Fuentes (2009; Montjour, 2011; Polanco, 2014 
el segmento reformulador reproduce el mismo conjunto de rasgos semánticos que el segmento reformulado. Desde el momento en que se usa una expresión alternativa, se produce un cambio en la formulación (no solo en el nivel formal, sino generalmente también en el plano semántico o pragmático) que entraña una cierta deformación del contenido de la expresión de partida (Fuchs, 1994). Por ello, la relación de equivalencia en una reformulación parafrástica es necesariamente gradual y puede oscilar entre una identidad semántico-conceptual elevada y una equivalencia semántica mínima, pero inferible en la medida en que la relación se establezca mediante un marcador cuyo valor semántico-pragmático apunte en una dirección de ( \pm ) equivalencia. Dicha equivalencia se funda en la interacción de las propiedades significativas compartidas entre A y A' (o inferidas contextualmente), en la instrucción del marcador que establece la relación (cuando este está presente) y en la reinterpretación contextual del nuevo enunciado ${ }^{5}$. En definitiva, toda reformulación constituye una actividad dinámica de construcción discursiva con la que el hablante trata de reconducir lo que ha dicho o quiere decir porque lo percibe como un problema de comunicación potencial.

Dado que toda reformulación implica una transformación potencial (una deformación del contenido de A y un distanciamiento del punto de vista enunciativo original más o menos evidentes), puede decirse que se establece lo que Fuchs (1994) denomina un "umbral de distorsión”, un espacio variable de interpretabilidad (de la equivalencia entre A y A') sujeto a límites también variables de aceptabilidad (relacionados con determinadas convenciones, los participantes discursivos y la situación de enunciación). Según Fuchs (1994: 18-19), en el curso del proceso de reformulación parafrástica, el hablante elabora un nuevo contenido A' a partir del contenido de A mediante la selección de determinados elementos enunciativos posibles. La elección de este contenido es tanto cuantitativa (puede expandir o reducir dicho contenido) como cualitativa (puede distanciarse más o menos del contenido original). Entre la hipotética identidad absoluta y el distanciamiento máximo que conforman los polos del continuum equivalencia-distanciamiento, el hablante dispone de un margen gradual de transformación del contenido de A que su interlocutor admitirá como aceptable mientras perciba dicha modificación dentro de los límites del umbral de distorsión. Los interlocutores se valen, en definitiva, de su conciencia metapragmática ${ }^{6}$, del conocimiento compartido que tienen sobre el uso de la reformulación y sus límites de interpretabilidad.

\section{La reformulación como construcción discursiva}

La reformulación, en tanto que estrategia discursiva, puede entenderse como un patrón discursivo convencional al que recurren los interlocutores durante el proceso dinámico de construcción-interpretación del discurso; esto es, como una construcción esquemática, abstracta y no especificada léxicamente (Goldberg, 1995; Ono y Thomson; 1995; Linell, 2009)7. Llevar

5 De ahí que sea preferible el término de dinámica de la identificación (Fuchs, 1994) al de predicación de identidad (Rossari, 1994) para referirse al tipo de operación reformuladora que llevan a cabo los marcadores parafrásticos.

6 Sobre el concepto de conciencia metapragmática véase Verschueren (2000, 2003) y Portolés (2004).

7 Aunque los conceptos de esquema y construcción están íntimamente relacionados, es preferible mantenerlos separados (Diessel, 2004). El concepto de construcción engloba tanto esquemas gramaticales abstractos, entre los que incluimos los discursivos (como sería el caso de los esquemas de reformulación que proponemos en este trabajo), como expresiones léxicas concretas, sintagmas léxicos o locuciones discursivas del tipo [pero vamos/vaya], [vamos que no] o [vaya que si___ (enunciado ecoico)] (Polanco, 2014; en prensa). En cambio, el concepto de esquema se aplica solo a esquemas o patrones gramaticales abstractos (por ejemplo, $\left[\mathbf{A}\right.$ reformulación $\left.\mathbf{A}^{\prime}\left(A \cong A^{\prime}\right)\right]$ para representar el esquema funcional de una reformulación parafrástica de equivalencia conceptual); es decir, un esquema puede concebirse como un tipo de construcción particular (Diessel, 2004). 
a cabo una reformulación implica activar un determinado marco interpretativo ${ }^{8}$ para dicha operación discursiva, que "obliga" al hablante a no transformar el segmento de partida más allá del umbral de distorsión $n^{9}$ asociado a dicho marco -incluso si el proceso se marca explícitamente con una partícula-, de manera que su interlocutor pueda interpretar el segmento reformulador (A') como una representación razonablemente equivalente de lo anterior (A).

En este sentido, el hablante elige una formulación determinada entre muchas posibles, la que en ese momento de la comunicación es más accesible, o le parece más pertinente, para reelaborar una formulación anterior, ante el supuesto de que no ha sido suficientemente clara. Y, para ello, el hablante utiliza un esquema discursivo que evoca un marco de interpretación concreto. Y por la misma razón, desde el momento en que el interlocutor reconoce la intención reformuladora del hablante, espera que la nueva formulación se ajuste a lo que él considera que puede ser una representación alternativa aceptable a partir del umbral de distorsión que este ha invocado y que ambos interlocutores comparten en mayor o menor medida.

Los interlocutores se valen, en definitiva, de su conocimiento metapragmático, esto es, del conocimiento global compartido que tienen sobre el uso de la reformulación (tanto los elementos formales como las condiciones para garantizar un uso adecuado) y sus límites de interpretabilidad (las restricciones semántico-pragmáticas asociadas al acto reformulador). Y es en este sentido en el que puede defenderse que el hablante recurre a un patrón discursivo asociado a una función discursiva prototípica convencionalizada, en el supuesto de que su interlocutor invocará un determinado marco de interpretación con su consecuente umbral de distorsión interpretativo asociado para interpretar la intención (re) formuladora del hablante.

\subsection{Los esquemas discursivos reformu- lativos}

Visto desde una perspectiva construccional, los esquemas discursivos expresan una función discursiva que activa un determinado marco de interpretación (a partir de ahora, MI). Este MI configura un bloque de información compuesto por un haz de rasgos formales, semántico-pragmáticos y prosódicos, que establece un umbral de distorsión interpretativa sobre el uso de la construcción-función discursiva y sobre el margen de variación en la interpretación.

Cada uso efectivo de estos esquemas abstractos prototípicos en la conversación o en el discurso, en los que se incluye un MD previsto prototípicamente por el MI de la construcción, representa una instancia concreta ${ }^{10}$ del esque-

8 Este concepto se desarrollará en el apartado 5 de este trabajo. Basamos el concepto de marco de interpretación (MI) en la noción de marco cognitivo (idealizado) o esquema de conocimiento ('knowledge schemata') que se maneja en Semántica Cognitiva. Estos conceptos se refieren principalmente al conocimiento implícito que subyace a la interpretación de las palabras conceptuales: "a word's meaning can be understood only with reference to a structured background of experience, beliefs or practices, constituting a kind of conceptual prerequisite for understanding the meaning" (Fillmore y Atkins, 1992: 76). No obstante, creemos que la noción de marco como conjunto de conocimiento estructurado ligado a una palabra o expresión más compleja (en distintos grados de esquematicidad o abstracción), conocimiento que incluye cómo usar una expresión determinada, puede aplicarse adecuadamente a los esquemas construccionales y a los marcadores del discurso y construcciones discursivas que estamos tratando en este trabajo. En este sentido, los marcos de interpretación recogen el hecho de que las experiencias discursivas previas forman parte del conocimiento general de base que los hablantes aportan al intercambio comunicativo, tanto como el conocimiento "cognitivo" inherente al concepto de frame (Lee, 2001: 190)

9 Recordamos que por umbral de distorsión (Fuchs, 1994) entendemos un espacio variable de interpretabilidad (de la relación entre los segmentos discursivos implicados en la (re)formulación) sujeto a límites también variables de aceptabilidad (relacionados con determinadas convenciones, los participantes discursivos y la situación de enunciación).

10 Las instancias del esquema general superordinado mantienen una relación relativa respecto de este y representan casos más o menos centrales de la construcción discursiva. Es decir, cada construcción discursiva puede presentar efectos prototípicos derivados de la relación del esquema construccional y sus instancias (Bybee, 1995). 
ma abstracto general. Así, por ejemplo, esquemas como

[A, es decir/o sea/vamos/vaya... A'], [A, en fin, en definitiva, es decir/o sea/vamos/vaya, (que) $\left.A^{\prime}\right]$ y [A, mejor dicho/más bien/vamos/ vaya... $\left.A^{\prime}\right]$

representan instancias de un esquema más general

[A REFORMULACIÓN A'].

En principio, un análisis como el que proponemos permitiría reconocer el estatus especial del sentido central o básico de la construcción, en nuestro caso, el de reformulación parafrástica de equivalencia conceptual para el esquema de reformulación [A REFORMULACIÓN $A^{\prime}\left(A \cong A^{\prime}\right)^{11}$ ]. Reformular, como hemos apuntado anteriormente, supone un volver a decir lo mismo de otra manera más ajustada a la intención comunicativa del emisor, y una manera de hacerlo consiste en repetir el mismo contenido, es decir, parafrasearIo. A medida que la "reformulación" se vaya alejando del contenido del segmento reformulado - esto es, en la medida en que A' deje de representar o comentar los mismos elementos tópicos que $\mathrm{A}$-, se producirá un alejamiento del centro categorial (la paráfrasis) y estaremos ante esquemas reformulativos cada vez menos típicos, menos esperables y, por tanto, menos interpretables como reformulaciones. Esto, por otra parte, es consecuente con una concepción gradual de la interpretación reformulativa: de una equivalencia conceptual amplia - valor prototípicoa una equivalencia mínima o nula, momento en el que podemos hablar de otro tipo de operación formulativa, la rearticulación. Un planteamiento como el anterior permite pensar en una especie de extensión interpretativa del concepto de reformulación (o lo que es lo mismo, de qué es reformular y de cuáles son sus límites) a partir del sentido central de paráfrasis conceptual.

Los esquemas construccionales anteriores pueden representarse como un inventario estructurado del conocimiento convencional que tiene un hablante sobre los recursos reformulativos de su lengua. Estos esquemas pueden encajarse dentro de un modelo con forma de red taxonómica que represente los distintos grados de generalidad entre los esquemas y sus instanciaciones más concretas, así como las distintas relaciones que se establecen entre los distintos nodos de la red (ver figura 1) 12. En función del principio de no sinonimia de las formas gramaticales (Goldberg, 1995), cada construcción con propiedades diferenciadas (de carácter fonológico, sintáctico, léxico, semántico o discursivofuncional) puede considerarse una construcción y se representa como un nodo independiente en la red construccional. Es razonable pensar, como tratamos de justificar en el apartado 4, que cada esquema de la red construccional responde a una conceptualización o una representación del conocimiento convencional diferenciado que tiene el hablante de los diferentes recursos reformulativos de su lengua.

Un modelo representacional de este tipo permite una categorización funcional flexible, dado que la relación entre funciones y esquemas funcionales afines se establece a partir de los distintos grados de representatividad que estos mantienen respecto de la función y del esquema generales; esto es, en función del grado de proximidad con el sentido central de la construcción general. La flexibilidad prevista por el modelo polisémico permite también establecer relaciones entre esquemas discursivos funcionalmente diferenciados. En un ejemplo como el siguiente:

11 Con el símbolo $\cong$ representamos la relación gradual de equivalencia conceptual asociada a este esquema construccional de forma prototípica.

12 Representamos con una línea continua las relaciones directas o prototípicas entre esquemas y construcciones, y con una línea discontinua las relaciones más indirectas o menos prototípicas. 


\section{FIGURA 1}

Red taxonómica de los esquemas discursivos asociados con la reformulación

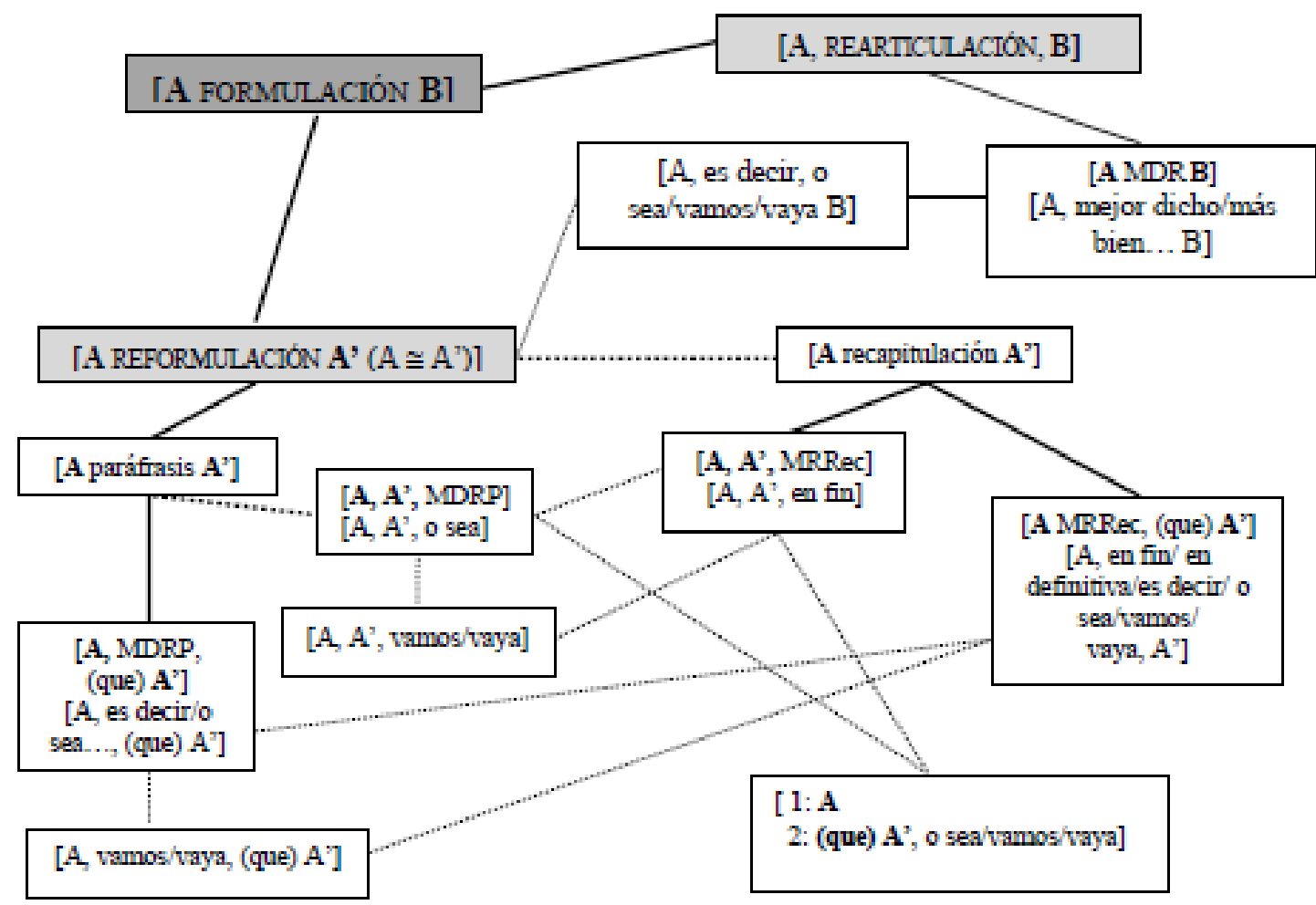

MDRP: MD de reformulación parafrástica

MIR: $M D$ de rectificación

MDRec: MD de recapitulación / conclusión

(4) Venga va aunque sean las tantas y sólo sea pa responder a Chelu, los amish son algo así como el "Pirulas" pero mucho más rústicos, vamos los tíos estos que van como los judíos ortodoxos, ya sabes en plan "skinetta". Sólo se alimentan de productos no manufacturados es decir los que se extraen directamente de las huertas, en las que trabajan con maquinaria primitiva, nada de M.C.I.A. y maquinaria pesada (como lo hacían nuestros tatarabuelos) y son muuuuuu respetuosos con el medio ambiente. En fin/Total/O sea/Vamos que [viven en el siglo XXI, pero actúan como si lo hiciesen a principios del XIX].

(http://es.groups.yahoo.com/group/fortunetellersclub/message/3254; en el original aparece el marcador vamos) la construcción [A, en fin/total/o sea/vamos/ vaya, (que) A'] es una instanciación del esquema discursivo superior [A RECAPITULACIÓN $A^{\prime}$ ], en tanto que $A^{\prime}$ reformula $A$ mediante un enunciado que recapitula o resume lo anterior. Por otro lado, dado que la recapitulación mantiene una vinculación tópica entre ambos segmentos, representa también una operación de reformulación menos prototípica que la paráfrasis, porque la relación de equivalencia conceptual puede resultar menos evidente.

Ahora bien, el esquema [A, o sea/vamos/ vaya, que $A^{\prime}$ ] también se utiliza para introducir un segmento $\left(A^{\prime}\right)$ que concluye el segmento previo (A) mediante la explicitación de una consecuencia derivada de este, como muestra el siguiente ejemplo: 
(5) He hablado con él y dice que tiene que acabar un trabajo para mañana y que va por la mitad. En fin/Total/O sea/Vamos/Vaya, que no viene.

En este sentido, la instancia construccional de reformulación recapitulativa, aun siendo una instancia prevista por la construcción discursiva reformulación [A REFORMULACIÓN $\boldsymbol{A}^{\prime}$ ] (relación que representamos en la figura 2 con una flecha continua), se vincula indirectamente con la construcción [A CONCLusión/CONSecuencia B] (relación que representamos con una flecha discontinua), en tanto que la operación de recapitulación puede consistir en la explicitación de una consecuencia. La recapitulación puede entenderse como una manera no prototípica de expresar la consecuencia que se deriva de un segmento discursivo previo.

Una concepción radial como la que hemos representado en las figuras 1 y 2 implica principalmente dos cosas:

a) La categorización de las funciones discursivas y de las construcciones o esquemas asociados no puede establecerse de manera discreta, sino a través de un criterio flexible que permita establecer unidades centrales (de activación inmediata) y unidades relacionadas con las anteriores en diferentes grados (es decir, construcciones más o menos prototípicas).

b) EI MD que puede aparecer en una construcción discursiva no puede definirse como el único portador del significado y de la función que caracteriza a la construcción discursiva, puesto que el contexto estructural (es decir, el esquema discursivo) también contribuye a la interpretación funcional.

Las construcciones discursivas esquemáticas son, pues, una conjunción de características formales y semántico-funcionales que definen la construcción en su conjunto. Funcionan como unidades simbólicas que aúnan forma y significado-función en un nivel de abstracción superior.

\subsection{Relación entre el esquema general y las instancias concretas}

Como hemos expuesto en el apartado anterior, las construcciones generales pueden instanciarse en construcciones concretas en las que el esquema general se materializa léxicamente (en nuestro caso, principalmente a través de la

\section{FIGURA 2}

Esquema de los grados de vinculación entre esquemas discursivos dentro de la red taxonómica

\section{[ $\boldsymbol{A}$ REFORMULACIÓN $\boldsymbol{A}^{\prime}$ ]}

$[\boldsymbol{A} \operatorname{MDRec} \boldsymbol{A}$ ]

[A, en fin, en definitiva, es decir/o sea/vamos/vaya, (que) A']

\section{[ $\boldsymbol{A}$ CONCLusión/CONsecuencia $\boldsymbol{B}$ ]}

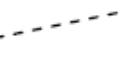

$[\mathrm{A}$, por tanto, en consecuencia, $\mathrm{B}] \quad[\mathrm{A}$, asi que/de

[A, de ahi que B (SV subj)]

MDRec: MD de recapitulación/conclusión 
inclusión de un MD). Asimismo, hemos apuntado que la relación entre los elementos que configuran la construcción concreta y el esquema general no es unidireccional, es decir, no viene dada únicamente por el valor del elemento léxico (el MD) que materializa el sentido o la función de la construcción. El significado del elemento léxico (la instrucción que establece el MD) funciona como un anclaje explícito de la función general que activa la construcción, pero no define necesariamente dicha función.

Esto último es fácil de advertir a partir de los distintos sentidos que puede cobrar un marcador discursivo como o sea o vamos en función del contexto en que aparece. Aunque estos marcadores pueden relacionarse con un significado de base estable ${ }^{13}$, que en buena medida persiste y condiciona la interpretación, la construcción discursiva ejerce también una considerable presión interpretativa, en tanto que establece un determinado marco de interpretación. En la medida en que el significado del MD y el procedimiento que active (esto es, su umbral de distorsión interpretativa) no entre en conflicto con el marco evocado por la construcción, la interpretación será posible, generalmente dentro de los límites establecidos tanto por la construcción como por el propio marcador. Así, en un ejemplo como el siguiente:

(6) Hay que informar, pero de forma gratis, es decir/o sea/vamos/vaya, que no les cueste a usted dinerillo al tener que ver esta revista, como en otras ocasiones. (CREA; Qué me dices, 19/10/96, Tele 5; en el original aparece vamos)

el contexto presenta un conjunto de rasgos característicos: comentario previo y posterior al MD completo, coorientación de los segmentos vinculados, relación de equivalencia conceptual entre ambos segmentos, posición medial de turno, entonación suspendida del MD. Este conjunto de rasgos activa, a su vez, un marco interpretativo prototípico concreto, el de reformulación parafrástica de equivalencia conceptual mediante aclaración o concreción. A esta interpretación contribuye también, obviamente, el marcador como elemento de relación prototípico previsto por la construcción para realizar la función prototípica de reformulación descrita, es decir, en tanto que anclaje explícito de la relación funcional entre ambos segmentos.

Ahora bien, que el MD explicite la relación funcional no significa que esta no pueda establecerse en su ausencia. Prueba de ello es que la función reformulativa puede llevarse a cabo sin marcador. Igualmente, si en ese mismo esquema apareciera algún elemento léxico ininterpretable o funcionalmente incompatible con la construcción, la interpretación por defecto seguiría siendo la misma, puesto que dicha interpretación sería la más coherente -y relevante-con el marco establecido por el esquema discursivo, tal como muestran los siguientes ejemplos:

(7) Hay que informar, pero de forma gratis <pausa> que no les cueste a usted dinerillo al tener que ver esta revista, como en otras ocasiones. (CREA; Qué me dices, 19/10/96, Tele 5; en el original aparece vamos).

(8) Hay que informar, pero de forma gratis, ajaedé, que no les cueste a usted dinerillo al tener que ver esta revista, como en otras ocasiones. (CREA; Qué me dices, 19/10/96, Tele 5; en el original aparece vamos).

A lo anterior podría objetarse que ese mismo contexto estructural, como muestra el ejemplo (9), puede ir asociado a otros significados, puede representar otras operaciones discursivas distintas, de modo que la estructura discursiva no

13 En el caso de o sea, un significado vinculado con la reformulación parafrástica de equivalencia conceptual; en el caso de vamos, con la formulación y la prosecución discursiva (Polanco, 2013a). 
se asocia con un significado concreto, sino que este debe especificarse a posteriori mediante un MD determinado, y que es este elemento el que confiere significado a la estructura:

(9) Hay que informar, pero [de forma gratis] ${ }_{A^{\prime}}$ o sea/vamos/vaya/en fin/mira/ya ves/(?)por tanto/(\#/*) en cualquier caso, [que no les cueste a usted dinerillo $]_{A^{*}}$

Sin embargo, no hay que olvidar que el uso de estos MD no es aleatorio, sino motivado. El hablante sabe de antemano qué clase de operación discursiva llevará a cabo (qué es lo que quiere hacer con su discurso) y, en consecuencia, escoge un esquema formulativo que le permita realizar dicha operación. En el ejemplo anterior, la relación entre los dos segmentos conectados es de semejanza conceptual $\left(A \cong A^{\prime}\right)$, de modo que el esquema propicio para dicha relación sería una reformulación parafrástica. El hablante sabe también que la función asociada prototípicamente a dicho esquema conlleva un determinado marco de interpretación (con su umbral de distorsión asociado); y que entre la información que contiene dicho marco se hallan determinadas piezas que transmiten explícitamente el tipo de función prevista por el esquema (por ejemplo, es decir, o sea o vamos). Este conocimiento lingüístico no es puntual, sino convencional o fuertemente generalizado. Y es un conocimiento compartido con un margen de error más o menos previsto por el marco de interpretación y el umbral de distorsión interpretativa.

Igualmente, no puede obviarse que entre las posibilidades discursivas para expresar una reformulación como la del ejemplo (6) hay varias opciones estructurales o esquemas discursivos vinculados en grados distintos de representatividad. Algunos esquemas, con el MD previsto por el esquema, serán una opción discursiva más afianzada, saliente y fácilmente activable que otros esquemas afines, dentro de un límite de interpretabilidad previsto por el marco de interpretación. Así, por ejemplo, los esquemas [A, es decir/o sea
$\left.A^{\prime}\right]$ y [A, en fin $\left.A^{\prime}\right]$ son opciones discursivas más accesibles que [A, vamos (que) $A^{\prime}$ ] para expresar las funciones reformulación parafrástica y reformulación recapitulativa, respectivamente.

Por otra parte, un MD prototípico para una función discursiva dada puede asociarse con otros esquemas discursivos como elemento no central. En estos casos, como muestra el ejemplo (4), que repetimos parcialmente en (10), el MD toma como sentido contextual el correspondiente al esquema discursivo en que aparece. De este modo, por ejemplo, cuando o sea o vamos aparecen en un contexto de reformulación recapitulativa, estos marcadores cobran automáticamente un sentido recapitulativo y cancelan su valor prototípico de reformulación parafrástica de equivalencia conceptual:

(10) (...) los amish (...). Sólo se alimentan de productos no manufacturados es decir los que se extraen directamente de las huertas, en las que trabajan con maquinaria primitiva, nada de M.C.I.A. y maquinaria pesada (como lo hacían nuestros tatarabuelos) y son muuuuuu respetuosos con el medio ambiente. O sea/ Vamos que viven en el siglo XXI, pero actúan como si lo hiciesen a principios del XIX.

(http://es.groups.yahoo.com/group/fortunetellersclub/message/3254)

El sentido recapitulativo que adquieren o sea 0 vamos en (10) es un sentido funcional derivado, obviamente, del contexto en que aparecen. Ahora bien, este sentido se activa por defecto siempre que estos marcadores aparecen en este tipo de esquema funcional, lo cual permite pensar que la interpretación concreta se deriva más bien como un valor generalizado (si no convencionalizado) y no como resultado de un reajuste interpretativo ad hoc a partir del contexto —opción esta última que, en estos casos al menos, no parece una opción cognitiva más económica. Es plausible asumir, por tanto, la existencia de una vinculación automática entre un marcador y el esquema discursivo en que aparece, de forma que el esquema 
discursivo activa por defecto uno de los valores del marcador a partir de una interpretación estereotípica del esquema discursivo.

Así, en un esquema como [A, vamos, que $\left.A^{\prime}\right]$, es razonable pensar que la lectura específica del valor asignado a vamos viene dada por la interpretación que se deriva por defecto del valor asociado estereotípicamente al esquema discursivo $\left[A, M D\right.$, que $\left.A^{\prime}\right]$, esto es, el de síntesis conceptual. Este valor estereotípico puede parafrasearse como

Se reformula con A' para extraer el elemento o los elementos esenciales de A (recapitular o resumir) o para explicitar una conclusión derivada de $A^{14}$.

El valor funcional de vamos en un contexto como el anterior puede tratarse, pues, no como una implicatura particularizada (como un sentido o inferencia ocasional), sino como una implicatura conversacional generalizada (Levinson, 2004 [2000]; ICG en adelante) implicitada por la forma del esquema discursivo [A, vamos, que $\left.A^{\prime}\right]$ y la aplicación de una heurística inferencial que restringe la interpretación del MD hacia la derivación de supuestos inmediatos sobre lo que es normal o estereotípicamente interpretable a partir del esquema discursivo en cuestión (Principio de I (informatividad) (Atlas y Levinson, 1981, apud Levinson, 2004 [2000]: 84) o principio I (Levinson, $2004[2001]))^{15}$

Si la relación entre el marcador y otros esquemas discursivos es frecuente, el marcador acaba por asumir la función asociada a la construcción, de modo que el MD amplía su ámbito de actuación y puede ir afianzando nuevos valores semánticos, como es el caso, por ejemplo, de vamos. De igual modo, la construcción discursiva amplía su marco de interpretación añadiendo nuevas variables de formalización con distintos grados de activación o prototipicidad, por ejemplo, ampliando la nómina de elementos discursivos que pueden saturar el hueco MD de la construcción con elementos no previstos inicialmente en el MI de la construcción, esto es, como elementos discursivos periféricos.

En definitiva, el esquema discursivo general establece las condiciones bajo las que una instancia concreta de la construcción será entendida como tal construcción, por ejemplo como reformulación parafrástica o como reformulación recapitulativa. Pero, a su vez, los MD que puedan aparecer en dicha construcción ayudan a concretar el significado funcional de la construcción en que aparecen. Dicho de otro modo, la relación entre el esquema más abstracto y sus diversas instanciaciones -y, por lo tanto, entre el significado más abstracto y los significados más concretos - es una relación condicionada de doble dirección: ascendente (de la construcción esquemática a la construcción concreta) y descendente (del significado del MD hacia el esquema discursivo).

\section{El conocimiento metapragmático de fondo: el marco de interpretación}

Hemos propuesto en el apartado anterior que tanto los esquemas construccionales como las construcciones discursivas y los MD se vinculan

14 Este valor asociado al esquema [A, MD, que A'] (y concretamente a sus instanciaciones, como [A, vamos, que A']) es, en nuestra opinión, un sentido construccional convencionalizado o, al menos, altamente generalizado (Polanco, 2013b). Este valor se deriva, probablemente, de la convencionalización de un inferencia-M generalizada por contraste entre el esquema anterior, que supone un esquema más complejo, y el esquema reformulador general [A, MD, A']. La introducción de la partícula que en el esquema genérico supone una opción marcada que implicita una interpretación no estereotípica del esquema resultante hacia el valor recapitulativo-conclusivo, que acaba convencionalizándose.

15 Igualmente, elementos como mira o ya ves, cuya función principal se asocia con tareas interactivo-discursivas y con valores fáticos y modales, adquieren valores reformulativos —es decir, no prototípicos - en contextos de reformulación parafrástica, como muestra el ejemplo (9) en cuerpo de texto. En estos casos, el marcador se reinterpreta contextualmente a partir del significado que emana del esquema discursivo en que se inserta. 
con un determinado marco de interpretación. En Semántica Cognitiva, las palabras (conceptuales) remiten a un dominio o marco ${ }^{16}$ conceptual compuesto de información diversa relacionada con el concepto representado por esa palabra. Este dominio conceptual se estructura en función de la experiencia que el hablante tiene del concepto, es decir, a partir de las relaciones que dicho concepto mantiene con otros conceptos afines en la realidad particular en que suele utilizarse.

Algo parecido puede decirse de las palabras gramaticales o no conceptuales. Estas palabras no evocan marcos conceptuales de la misma manera que las palabras conceptuales, en tanto que no remiten a un conjunto de conocimientos y experiencias relacionados con conceptos del mundo. Pese a ello, no dejan de evocar algún tipo de marco o dominio sobre el que interpretarlas. El dominio significativo de estas palabras también recoge la experiencia del hablante respecto de ellas, es decir, información relacionada con los usos y las interpretaciones de esos usos. Esta información, asimismo, debe de estar ordenada de alguna manera, normalmente en relación con el grado de saliencia o prioridad de activación, dado el conocimiento que el hablante tiene de dichos elementos. Este conocimiento puede variar de un hablante a otro, pero debe haber, sin duda, una parte del dominio compartido que permite la interpretación.

En el caso que nos ocupa, el marco de interpretación recoge el conocimiento metapragmático de fondo que poseen los hablantes acerca del uso-interpretación de los elementos discursivos que conforman su gramática mental. El marco de interpretación representa un conjunto de información estructurado y convencional (o con un grado de estabilidad elevado) compuesto por un haz de rasgos sintácticos, semántico-pragmáticos y prosódicos, que establece un umbral de distorsión interpretativa sobre el uso de la construcción-función discursiva y sobre el margen de variación en la interpretación. En este sentido, siguiendo a Lee (2001: 190), estimamos que el concepto cognitivo de marco, entendido como trasfondo estructurado de experiencias, creencias o prácticas que condicionan "conceptualmente" la aprehensión del significado (Fillmore y Atkins, 1992: 76), debe ampliarse para dar cuenta del hecho de que las experiencias discursivas previas, con toda la información relevante asociada a tales experiencias repetidas, también forman parte del conocimiento convencional de base que los hablantes aportan al intercambio comunicativo - en la misma medida en que el conocimiento "cognitivo" inherente al concepto de frame guía la interpretación de las expresiones conceptuales.

Como hemos apuntado en 3.2., cada construcción activa un marco de interpretación concreto, con un cierto margen de flexibilidad interpretativa en función del grado de abstracción que presente la construcción. El marco de interpretación especificará no solo la información sintáctica y semántico-pragmática prototípica asociada a la construcción (especificidad que variará en función del grado de abstracción del esquema construccional), sino también los elementos discursivos que pueden figurar prototípicamente en tal construcción, es decir, que pueden saturar el hueco MD previsto por la construcción. El MD, por su parte, también se asocia con un marco de interpretación que recogerá la información convencional (o fuertemente afianzada) que establece las condiciones prototípicas de uso del marcador (Polanco, 2013, 2014). Cada

16 Según Fillmore, "by the term 'frame' I have in mind any system of concepts related in such a way that to understand any one of them you have to understand the whole structure in which it fits" (Fillmore, 1982: 111). Así pues, un marco (frame) es un cuerpo coherente de conocimiento presupuesto por un concepto léxico (word concept) (Croft y Cruse, 2004: 17).

Un marco o dominio es una estructura semántica que funciona como base (que recoge el conocimiento o estructura conceptual que está presupuesta por el concepto perfilado) para por lo menos un concepto perfilado (el perfil se refiere al concepto simbolizado por la palabra en cuestión) (Croft y Cruse, 2004: 15). 
marco, que representaremos en tablas en el apartado 5 , incluye rasgos formales y semánticopragmáticos de nivel y naturaleza distintos, asociados convencionalmente o de manera estable al uso e interpretación del MD en un esquema construccional.

El significado del MD puede especificar la interpretación de la construcción, pero no la determina necesariamente. Así, en un esquema discursivo de reformulación recapitulativa se activa una interpretación por defecto de síntesis o condensación informativa, en tanto que el segmento reformulador A' cierra el segmento de información previo, recapitulándolo o explicitando alguna conclusión derivada del segmento previo. La inclusión de un marcador como en fin aporta una especificación semántica a partir de su propio significado de finalización (de punto final), que refuerza la interpretación recapitulativa de la construcción; es decir, el marcador ayuda a especificar la interpretación, pero no la establece per se. Dicho de otro modo, el esquema discursivo activará por defecto una interpretación vinculada a la operación discursiva que se establece entre los componentes del discurso que saturan el esquema (es decir, en función de la relación semántico-pragmática que se establece entre $A$ y $A^{\prime}$ ). El MD, por su parte, reforzará dicha interpretación si constituye un elemento prototípico previsto por la construcción (si es un elemento central de esta). En caso contrario, y a no ser que el significado del MD sea incompatible con la construcción, el marcador recibirá por defecto la interpretación establecida por la construcción. Esto, por un lado, permite explicar por qué un MD no prototípico de determinada función-construcción (por ejemplo, o sea o vamos para expresar conclusión-recapitulación) puede interpretarse con una función asociada a la construcción, aunque el MD no sea un elemento central de esta; $y$, por otro, permite ver que la polifuncionalidad y polisemia de palabras como vamos o vaya (y de otras partículas polifuncionales) es, en buena medida, una función de la relación entre el elemento discursivo y las construcciones de las que puede formar parte (Polanco, 2013a, 2014).

Esta doble especificación informativa, tanto en el marco de interpretación del esquema discursivo como del marcador, supone, en principio, una sobreespecificación que algunos modelos de corte minimalista (entre ellos, algunos modelos construccionales) tratan de evitar mediante el recurso al contexto y a la inferencia. En algunos modelos construccionales, como la Gramática de Construcciones (Fillmore y Kay, 1993; Kay y Fillmore, 1999), las construcciones de nivel inferior (low-level constructions) - las instanciaciones de una construcción de nivel esquemático superior - suelen estar subespecificadas, en tanto que solo incluyen la parte de la información que no está presente ya en representaciones más esquemáticas. En estos modelos teóricos, llamados de herencia completa (Goldberg, 1995: 73-74), la información se representa de forma no redundante y se hereda; es decir, se parte de la base de que las representaciones gramaticales son máximamente económicas y no redundantes.

Así, por ejemplo, según este modelo representacional, el esquema recapitulativo $\left[A_{\gamma^{\prime}}\left(A_{\nu^{\prime}} A_{n}\right)\right.$ <pausa> vamos (<pausa〉) que $A^{\prime}$ ] no incluiría la información sintáctica o semántico-pragmática que puede recuperarse a partir de un esquema más abstracto [A <pausa> reformulación parafrástica <pausa> A'], que a su vez "hereda" información de un posible esquema superordinado [A REFORMULACIÓN A']. En los modelos construccionales de herencia completa, las construcciones de nivel inferior contienen solo la información que no comparten con otras construcciones. En consecuencia, la representación del marco de interpretación se reduce al mínimo: cada parte de la información relevante para el uso e interpretación de las construcciones se representa solo en una parte de la gramática mental (Diessel, 2004).

En este trabajo, sin embargo, seguimos un modelo de representación basado en el uso, modelo que permite representar la información en distintos niveles de la jerarquía taxonómica construccional, de modo que las construcciones de nivel inferior pueden estar completamente 
especificadas (Goldberg, 1995: cap. 3). Este modelo se denomina de entrada completa (Goldberg, 1995). Las construcciones afianzadas que presentan un nivel de activación elevado, independientemente del nivel de esquematicidad que presenten, despliegan toda la información necesaria para la interpretación, incluyendo la información que puedan compartir con otras construcciones de un nivel más abstracto. Por tanto, las construcciones de nivel inferior no heredan información necesariamente desde otros niveles superiores, sino que se relacionan con representaciones más esquemáticas mediante diferentes vínculos de instanciación, entre los que se incluye la superposición de información; esto es, la misma información puede almacenarse de forma redundante en diferentes niveles de abstracción y no necesariamente en el nivel más abstracto.

Un modelo de herencia completa minimiza el almacenamiento de información redundante, pero incrementa el procesamiento en línea para poder acceder a la información necesaria para la generación e interpretación de los enunciados. El modelo de entrada completa, en cambio, minimiza el procesamiento en línea durante la producción e interpretación de enunciados, permitiendo que pueda almacenarse tanta información como sea necesaria (y posible) en distintos niveles de activación (Goldberg, 1995: 74). Esto no significa que el modelo de entrada completa sea siempre preferible, sino que representa una opción compatible con el modelo de herencia completa. Evidentemente, los hablantes no almacenan una representación de todos los enunciados que pueden formular o escuchar; antes bien, generalizan a partir de las categorías implicadas en esos enunciados, formando esquemas de distinto nivel de abstracción (Croft y Cruse, 2004: 278). No obstante, la tendencia a la generalización no supone necesariamente que los hablantes tiendan siempre hacia la parsimonia y la elegancia en la representación del conocimiento gramati$\mathrm{cal}^{17}$.

El modelo de herencia completa puede ser, en principio, una opción adecuada para describir, por ejemplo, dos de las instanciaciones del esquema reformulación [A, REFORMULACIÓN A'], a saber: la reformulación explicativa (en la que puede incluirse la reformulación de concreción o matización) y la reformulación recapitulativa (principalmente cuando el esquema presenta una relación entre dos segmentos simples $A$ y $\left.A^{\prime}\right)^{18}$. En estos casos, puede argüirse que los esquemas más concretos reciben una especificación del esquema más abstracto hacia un sentido relacionado con el sentido central de la construcción, el de reformulación parafrástica de equivalencia conceptual. Este sentido central viene a representar de forma prototípica la función de reformular tal como la entendemos en este trabajo, es decir, como un volver a decir de otro modo lo que ya se ha dicho. La interpretación concreta se derivará de la presión que ejerza el marcador concreto que se emplee en cada construcción más un (re) ajuste contextual ${ }^{19}$

En definitiva, mientras que en un modelo de herencia completa la información fluye durante el procesamiento hacia abajo a partir de las construcciones más esquemáticas, en el modelo

17 De hecho, como sugiere Barsalou, hay evidencias psicológicas que avalan la representación redundante de información: "concepts and properties in human knowledge are organized with little concern for elegance and parsimony" (1992: 180).

18 Cuando se establece una relación de reformulación recapitulativa entre un segmento complejo [ $\left.A_{\gamma}\left(A_{\gamma} A_{n}\right)\right]$ y un segmento sintetizador simple A', podría hablarse de un esquema con especificaciones distintivas diferentes del esquema simple [A, A']; es decir, podría tratarse como una construcción discursiva distinta.

19 Una explicación alternativa es entender que cada esquema construccional diferente conlleva también un significado distinto, de manera que la especificación semántica no vendría dada inferencialmente, sino a partir de la propia construcción. Una posible evidencia de esto, como hemos tratado de demostrar en el apartado anterior, es el hecho de que el hueco MD en cada esquema discursivo puede rellenarse con distintos marcadores con significados diferentes, sin que ello altere el significado global que emana del conjunto. 
de entrada completa el procesamiento implica la activación de las construcciones más afianzadas cuyo marco de interpretación se ajuste mejor a la intención comunicativa del hablante y a las estructuras utilizadas para vehicular dicha intención.

Las construcciones más específicas se ajustan con mayor exactitud a lo que enuncian efectivamente los hablantes (es decir, a los enunciados emitidos), de modo que estas se activarán con mayor frecuencia que las más abstractas. En consecuencia, es plausible admitir que su interpretación puede realizarse directamente sobre un marco de interpretación informativamente completo asociado directamente al esquema específico en cuestión, y no necesariamente a partir de esquemas superordinados mediante derivación inferencial.

Así, en nuestra opinión, parece más razonable pensar que la interpretación de una secuencia enunciativa como

(11)Faltó la mitad de la gente, la comida era malísima, se quedaron cortos con la bebida y encima se puso a llover; vamos, que la fiesta fue un desastre

activa directamente el esquema $\left[A_{1}\left(A_{2}, A_{n}\right)\right.$, vamos, que $A^{\prime}$ ] y convoca un marco de interpretación especificado que se vincula con esa construcción, a saber, con la función de reformulación recapitulativo-conclusiva. En cierto modo, la interpretación de este esquema podría interpretarse como una implicatura conversacional generalizada a partir de la presencia de la partícula que, la cual modaliza el enunciado recapitulador y aporta una interpretación marcada respecto del mismo esquema sin dicha partícula. Ahora bien, el esquema $\left[A_{1}\left(A_{2}, A_{n}\right)\right.$, vamos, que $\left.A^{\prime}\right]$ presenta una restricción sintáctica ausente en el esquema sin la partícula [A, ref. recap., $\left.A^{\prime}\right]$ : el segmento recapitulador tiene que ser un constituyente proposicional completo (una oración o un elemento interjectivo). Esta diferencia sintáctica, más el hecho de que este esquema presente una alta frecuencia de uso, permite pensar no solo en un grado de afianzamiento elevado del esquema, sino en un esquema distinto con especificaciones formales y semántico-pragmáticas distintas ${ }^{20}$.

\section{Aplicación del modelo construccional a los valores parafrásticos de vamos}

En este apartado, aplicaremos el modelo construccional que hemos presentado a los valores de reformulación parafrástica (equivalencia conceptual) del marcador vamos.

Tanto los esquemas construccionales (por ejemplo, el esquema de reformulación parafrástica $\left.\left[A, M D, A^{\prime}\left(A \cong A^{\prime}\right)\right]\right)$ como las construcciones discursivas (por ejemplo, $\left[A_{1}\left(A_{2}, A_{n}\right)\right.$, vamos, que $\left.A^{\prime}\right]$ ) y los MD se vinculan con un determinado marco de interpretación. Así, la función discursiva reformulación, con un esquema prototípico $\left[A, M D, A^{\prime}\right]$, activa un marco de interpretación (MI) subespecificado en el que figura la información básica sobre el uso convencional asociado con la función prototípica de "volver a decir A", que activará una interpretación por defecto hacia el sentido de paráfrasis de equivalencia conceptual (sentido central del esquema construccional) (ver tabla 1$)$.

La inclusión en el esquema discursivo anterior de un marcador como vamos activará el sentido formulativo de este marcador (Polanco, 2013a: 232-238), dado que este es el valor semántico que se ajusta a las condiciones contextuales previstas en el MI del marcador. Ahora bien, el sentido formulativo solo apunta hacia una interpretación metadiscursiva en sentido am-

20 En este punto, seguimos el principio de no sinonimia de las formas gramaticales planteado por Goldberg (1995: 367), según el cual la diferencia en la forma lingüística supone una diferencia semántica y/o pragmática. 


\section{TABLA 1}

MI del esquema discursivo de reformulación parafrástica

\begin{tabular}{|c|c|c|c|}
\hline & \multicolumn{3}{|c|}{$\begin{array}{l}\text { Marco de interpretación del esquema discursivo } \\
\text { de reformulación parafrástica }\end{array}$} \\
\hline $\begin{array}{l}\text { Esquema discursivo } \\
\text { REFORMULACIÓN }\end{array}$ & \multicolumn{3}{|c|}{$\mathbf{A}<$ pausa $>\mathbf{M D}<$ pausa $>\mathbf{A}^{\prime}$} \\
\hline INFO. SINTÁCTICA & \multicolumn{3}{|c|}{$\begin{array}{l}\text { Las unidades discursivas A y A' deben ser enunciados completos sintáctica y } \\
\text { semánticamente. }\end{array}$} \\
\hline INFO. SEMÁNTICA & \multicolumn{3}{|c|}{ Se establece una relación de equivalencia conceptual entre $A$ y $A^{\prime}\left(A \cong A^{\prime}\right)$. } \\
\hline $\begin{array}{l}\text { FUNCIÒN PRAGMÁTICO- } \\
\text { DISCURSIVA }\end{array}$ & \multicolumn{3}{|c|}{$\begin{array}{l}\text { Reformular un segmento discursivo A mediante otro segmento A' que aclara } \\
\text { algún elemento del segmento previo (a través de una expansión o reducción } \\
\text { informativa). }\end{array}$} \\
\hline \multirow{5}{*}{$\begin{array}{l}\text { ELEMENTO DE UNIÓN } \\
\text { ENTRE A Y A' }\end{array}$} & \multicolumn{3}{|c|}{ Yuxtaposición: pausa marcada entre A y A’. } \\
\hline & \multicolumn{3}{|c|}{ Yuxtaposición: marcación prosódica del segmento reformulador A’. } \\
\hline & \multirow{3}{*}{$\begin{array}{l}\text { MD } \\
\text { REFORMULADOR }\end{array}$} & $\begin{array}{l}\text { Elemento léxico } \\
\text { (de más a menos } \\
\text { prototipicidad) }\end{array}$ & $\begin{array}{l}\text { Es decir, o sea, en otras palabras, } \\
\text { dicho de otro modo, esto es, (...), } \\
\text { vamos, vaya, en fin, en definitiva, } \\
(\text { (...), mejor dicho, más bien, (...). }\end{array}$ \\
\hline & & $\begin{array}{l}\text { Ubicación en } \\
\text { el esquema } \\
\text { discursivo }\end{array}$ & $\begin{array}{l}\text { Antepuesto al segmento } \\
\text { reformulador } A^{\prime} \text {. }\end{array}$ \\
\hline & & Info. prosódica & $\begin{array}{l}\text { Entonación suspendida o } \\
\text { descendente. }\end{array}$ \\
\hline
\end{tabular}

plio, de modo que la instrucción de equivalencia conceptual entre el segmento que introduce y el segmento previo se debe a una reinterpretación contextual del valor formulativo. Esta interpretación más específica viene dada por el sentido prototípico de la construcción discursiva en la que se inserta vamos, a saber, el de reformulación parafrástica de equivalencia conceptual. En este sentido, puede defenderse que el valor concreto de reformulación parafrástica del marcador se interpreta por defecto siempre que este se utilice en un contexto funcional $\left[A, M D, A^{\prime}\right]$ y no haya información contextual que oriente en otra dirección interpretativa, en función de una heurística interpretativa basada en el principio de Informatividad: "lo que se expresa de forma prototípica se interpreta de forma prototípica” (Levinson, 2004 [2000]). Esta heurística podría parafrasearse como:
Si un MD inespecífico se incluye en una construcción discursiva $X$, con un marco de interpretación Y, el MD se interpretará por defecto con la función especificada en dicho marco de interpretación en función del principio I (informatividad) a menos que haya información contextual que indique lo contrario.

De este modo, cuando un marcador con un valor metadiscursivo tan inespecífico como vamos se utiliza como elemento de unión en un esquema como el de reformulación parafrástica que estamos tratando, el valor funcional previsto en el MI de ese esquema discursivo pasa a constituir automáticamente el sentido funcional específico del marcador en ese contexto funcional. El esquema discursivo resultante, que incluye un MD concreto (en este caso, vamos), representa una instancia del esquema discursivo superor- 
dinado $\left[A, M D, A^{\prime}\right]$, de manera que el esquema más concreto hereda la información prevista en el MI del esquema más abstracto. La operación interpretativa anterior puede resumirse en una ecuación del tipo

[MD polisémico o subespecificado + esquema discursivo $X=$ función específica del MD].

El marco de interpretación del esquema discursivo especifica no solo la información sintáctica y semántico-pragmática prototípica asociada a ese esquema, sino también los elementos discursivos que pueden figurar prototípicamente en tal construcción. Por su parte, el MD también se asocia con un marco de interpretación que recoge la información convencional (o fuertemente afianzada), que establece las condiciones prototípicas de uso del marcador, en el caso que nos ocupa, el uso parafrástico de vamos.

Así, pues, la red semántica de vamos se configura como una estructura de conocimiento compleja que alberga información diversa acerca de lo que el hablante sabe sobre el uso de ese elemento lingüístico (Polanco, 2013a). La infor- mación contenida en esa red experiencial, que conforma lo que el hablante sabe acerca de vamos, se estructura, a su vez, en distintas parcelas de conocimiento más específico (los marcos de interpretación) en los que figura la información relevante asociada a los usos concretos de ese elemento.

Las siguientes tablas son un intento de representación de los marcos de interpretación de vamos asociados con la reformulación parafrástica.

La tabla 2 representa el contexto de reformulación más prototípico, en el que vamos se sitúa en posición medial de intervención, introduciendo el segmento reformulador.

La tabla 3 representa un esquema de reformulación parafrástica menos prototípico que el anterior, en tanto que el marcador discursivo aparece pospuesto al segmento reformulador.

La tabla 4 representa un contexto funcional de heterorreformulación asociado prototípicamente con la reformulación recapitulativoconclusiva. No obstante, la interpretación construccional de recapitulación puede diluirse o cancelarse si la relación entre los segmentos relacionados es de equivalencia conceptual.

\section{TABLA 2}

Marco de interpretación de vamos en contextos monológicos de reformulación parafrástica

\section{Marco de interpretación de vamos en contextos monológicos} de reformulación parafrástica

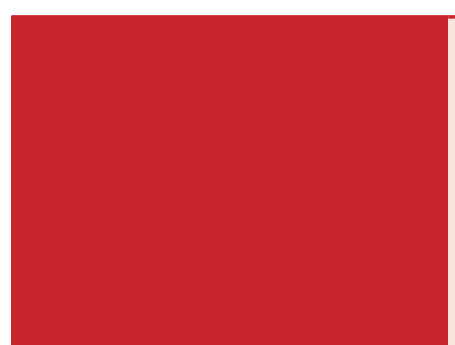

$\mathbf{A}\left\langle\right.$ pausa $>$ vamos $\left\langle\right.$ pausa $>\mathbf{A}^{\prime}\left(A=A^{\prime}\right)$

ESQUEMA DISCURSIVO
"En su momento, hubo tesis por las cuales el segundo canal iba a ser un canal que se podría haber financiado sin publicidad, vamos, a costa del presupuesto." (CREA; Debate: Ias nuevas televisiones, 10/12/87, TVE 1).

(Esquema prototípico de reformulación parafrástica. La interpretación por defecto de equivalencia conceptual puede cancelarse si la relación entre A y A’ no es de equivalencia conceptual $\left.\left(A \neq A^{\prime}\right)\right)$.

"sacaba la silla y allí la he visto yo más de un año, sentada en la silla; vamos, de soltera, que, ya después de casada, no” (Fuentes 1998: 180)

[El segmento que introduce vamos no es una paráfrasis de lo anterior, sino un cambio de perspectiva enunciativa con el que se resitúa contextualmente al oyente introduciendo nuevos datos ("de soltera, que ya, de después de casada, no") que ayudan a comprender mejor la intención comunicativa del hablante; se trata de un uso rearticulador] 


\begin{tabular}{ll}
\hline INFO SINTÁCTICA & $\begin{array}{l}\text { Entre unidades discursivas completas sintáctica y semánticamente que mantienen } \\
\text { entre sí una relación de equivalencia conceptual. } \\
\text { Aparece antepuesto al enunciado reformulador. }\end{array}$ \\
\hline UBICACIÓN EN EL TURNO & Posición medial de intervención y/o turno. \\
\hline INFO PROSÓDICA & Entonación suspendida o descendente. \\
\hline $\begin{array}{l}\text { FUNCIÓN } \\
\text { PRAGMÁTICO-DISCURSIVA }\end{array}$ & $\begin{array}{l}\text { Introducir un enunciado que aclara algún elemento del segmento previo (mediante } \\
\text { una expansión o reducción informativa). }\end{array}$ \\
\hline
\end{tabular}

\section{TABLA 3}

Marco de interpretación de vamos en contextos marcados de reformulación parafrástica

\section{Marco de interpretación de vamos en contextos marcados} de reformulación parafrástica

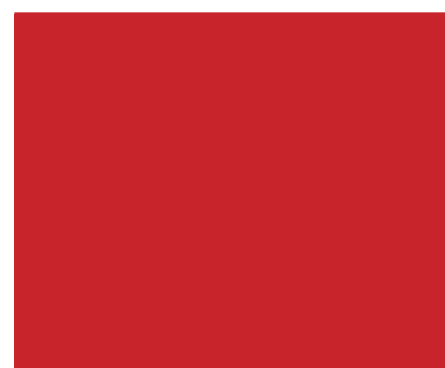

ESQUEMA DISCURSIVO

\section{$\mathbf{A}<$ pausa $>\mathbf{A}^{\prime}>$ pausa $>$ vamos}

"Yo trabajaba para uno del poblado, un tío al que llamábamos Lite, que había sido lejía y debía tener veintitrés o veinticuatro años o menos, pero que era mayor, hombre ya, vamos, porque ya había hecho el servicio militar, en los lejías, como ya he dicho." (CREA; EI Mundo, 15/01/1995: JUAN MADRID. MEMORIAS DE UN ASESINO (II). La muerte es mi compañera).

(Esquema no prototípico de reformulación parafrástica; la posición pospuesta del MD genera también una ICG de intensificación ilocutiva y refuerzo argumentativo La interpretación por defecto de refuerzo ilocutivo se sumará a la interpretación parafrástica si la relación entre A y A' es de equivalencia conceptual ( $\left.A=A^{\prime}\right)$.

Por el contrario, la ICG de refuerzo ilocutivo primará sobre la lectura parafrástica, cancelándola, si no hay una relación de equivalencia conceptual entre los segmentos relacionados $\left(A \neq A^{\prime}\right)$.)

"Ya no se puede creer en ningún partido solo, sino que tienes que pensar que para mí hay hipócritas en ambos bandos, se ha visto, siempre, vamos. Se está viendo ahora mucho y ya está. Nada más que eso." (CREA; La Luna, 05/09/89, TVE 1).

Tras una unidad discursiva completa sintáctica y semánticamente que mantiene una relación de equivalencia conceptual con otra unidad discursiva anterior de las mismas características.

Aparece pospuesto al enunciado reformulador.

\section{UBICACIÓN EN EL TURNO}

INFO PROSÓDICA

FUNCIÓN PRAGMÁTICO-DISCURSIVA
Posición medial o final de intervención/turno.

Entonación suspendida o descendente.

Cerrar un segmento (reformulador) que aclara algún elemento del segmento previo, apuntando retroactivamente hacia lo enunciado.

\section{Conclusión}

En este trabajo, hemos presentado la potencialidad explicativa que puede tener un tratamiento construccional de los contextos funcionales en la descripción semántico-funcional de elemen- tos discursivos funcionalmente complejos como los marcadores conversacionales.

Además de los sentidos convencionales asociados a un marcador, este suele activar determinados sentidos contextuales que parecen interpretarse por defecto, añadidos al significado 


\section{TABLA 4}

Marco de interpretación de vamos en contextos dialógicos no prototípicos de reformulación parafrástica

\begin{tabular}{|c|c|}
\hline & $\begin{array}{c}\text { Marco de interpretación de vamos en contextos dialógicos no prototípicos } \\
\text { de reformulación parafrástica }\end{array}$ \\
\hline ESQUEMA DISCURSIVO & 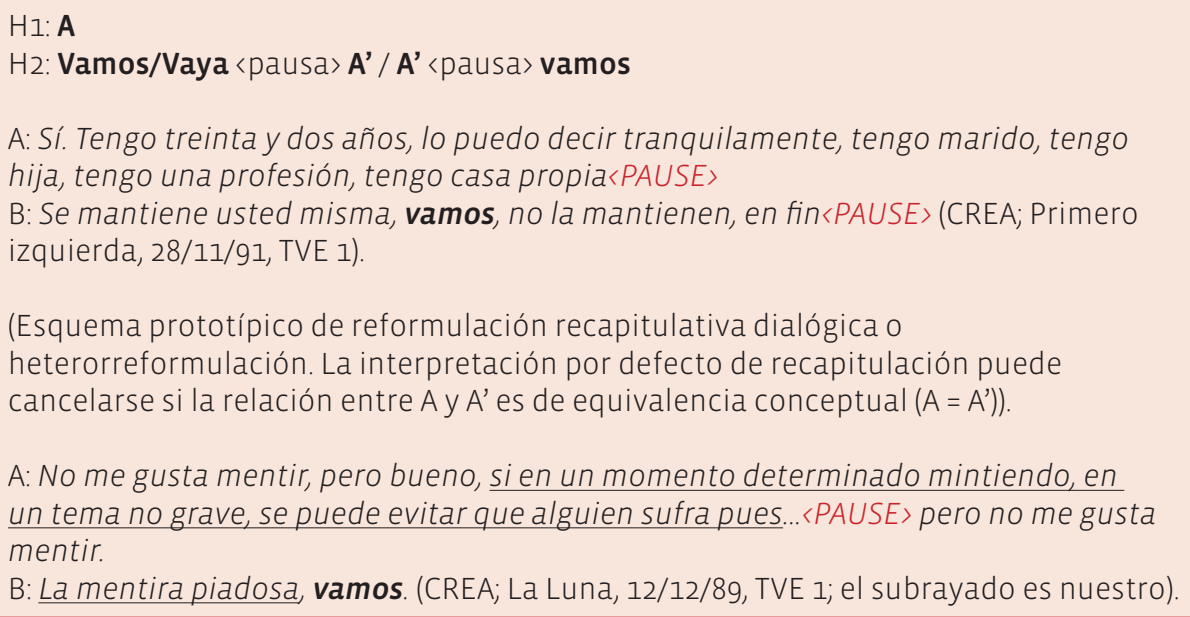 \\
\hline INFO SINTÁCTICA & $\begin{array}{l}\text { Entre unidades discursivas completas sintáctica y semánticamente, en intervenciones } \\
\text { y turnos diferentes ( } \mathrm{H} 1 \text { y } \mathrm{H}_{2} \text { ) que mantienen entre sí una relación de equivalencia } \\
\text { conceptual. El MD introduce el segmento reformulador en la intervención reactiva } \\
\text { (H2). } \\
\text { Tras una unidad discursiva completa sintáctica y semánticamente }\left(\mathrm{H}_{2}\right) \text { que mantiene } \\
\text { una relación de equivalencia conceptual con la unidad discursiva de las mismas } \\
\text { características ( } \mathrm{H} 2 \text { ) de la intervención inmediatamente anterior. El MD aparece } \\
\text { pospuesto al enunciado reformulador en la intervención reactiva (H2). }\end{array}$ \\
\hline UBICACIÓN EN EL TURNO & Posición inicial o medial-final de intervención/turno. \\
\hline INFO PROSÓDICA & Entonación suspendida o descendente. \\
\hline $\begin{array}{l}\text { FUNCIÓN } \\
\text { PRAGMÁTICO-DISCURSIVA }\end{array}$ & $\begin{array}{l}\text { Introducir un segmento (reformulador) que aclara algún elemento del segmento } \\
\text { previo; o cerrarlo, apuntando retroactivamente hacia lo enunciado. }\end{array}$ \\
\hline
\end{tabular}

nuclear, cuando este elemento forma parte de ciertos contextos funcionales prototípicos o esquemas funcionales. Estas interpretaciones funcionales más específicas son el producto de la interrelación entre un determinado sentido básico o convencional y ciertos esquemas discursivos prototípicos y están fuertemente motivadas por esa interrelación. Con todo, pese a su "fijación" semántico-cognitiva, no son sentidos convencionales. Estos sentidos, que hemos denominado construccionales, pueden concebirse como lecturas por defecto, en tanto que se activan automáticamente en función de dicha interrelación. Y son también, al mismo tiempo, interpretacio- nes cancelables, esto es, interpretaciones que pueden anularse si existe presión contextual en una dirección interpretativa distinta.

Para el tratamiento de los sentidos construccionales, hemos propuesto un acercamiento a la interpretación de estos valores contextuales que aúna la descripción gramatical y semánticodiscursiva desde una perspectiva construccional basada en el uso y la Teoría de las implicaturas conversacionales generalizadas (Levinson, 2004 [2000]). Con la incorporación de las ICGs a la descripción semántico-pragmática de marcadores altamente polifuncionales, como son los marcadores conversacionales, puede explicarse mejor, 
en nuestra opinión, la intuición de que algunas interpretaciones discursivas e interjectivas de estos elementos son constantes en determinados contextos semántico-estructurales o esquemas construccionales, en la medida en que estos contextos inducen "por defecto" una interpretación funcional ligada exclusivamente a ese contexto.

\section{Bibliografía citada}

Barsalou, Lawrence, 1992: Cognitive Psychology: an overviewfor cognitive scientists, Hillsdale, NJ: Lawrence Erlbaum Associates.

Briz, Antonio, Salvador Pons y José Portolés (coords.), 2008: Diccionario de partículas discursivas del español [www.dpde.es, fecha de consulta: 20 de noviembre de 2014].

Bybee, Joan, 1995: "Regular morphology and the lexicon", Language and Cognitive Processes 10, 425-455.

Calsamiglia, Helena y Amparo Tusón, 1999: Las cosas del decir, Barcelona: Ariel.

CoRtés, Luis y Ma Matilde CAmacho, 2005: Unidades de segmentación y marcadores del discurso, Madrid: Arco Libros.

Croft, William y Alan Cruse, 2004: Cognitive Linguistics, Cambridge: Cambridge University Press.

Diessel, Holger, 2004: "A dynamic network model of grammatical constructions" en Holger DiesSEL: The Adquisition of Complex Sentences, cap. 2, Cambridge: Cambridge University Press, 1340, [http://www.personal.unijena.de/ x4diho/ The\%20usage\%2obased\%20model.pdf, fecha de consulta: 2 de septiembre de 2014].

Fillmore, Charles, 1982: "Frame Semantics" en The Linguistic Society of Korea (eds.): Linguistics in the morning calm, Seúl: Hanshin, 111-137.
Fillmore, Charles y Sue ATKINS, 1992: "Towards a Frame-Based Lexicon: the Semantics of Risk and its Neighbors" en Adrienne LeHrer y Eva KitTAY: Frames, Fields and Contrasts: New Essays in Semantic and Lexical Organization, Hilldale: Lawrence Erlbaum, 75-102.

Fuentes Rodriguez, Catalina, 1998: "Vamos: un conector de gran complejidad" en $M^{a}$ Antonia MARtín Zorraquino y Estrella Montolio Durán (eds.): Los marcadores del discurso. Teoría y análisis, Madrid: Arco Libros, 177-192.

Fuentes Rodriguez, Catalina, 2009: Diccionario de conectores y operadores del español, Madrid: Arco Libros.

Fuchs, Catherine, 1994: Paraphrase et énonciation, Paris: Ophrys.

Garcés, Ma Pilar, 2005: "Reformulación y marcadores de reformulación” en Manuel Casado Velarde, Ramón González Ruiz y Ósar Loureda Lamas (eds.): Estudios sobre lo metalingüístico (en español), Frankfurt: Peter Lang, 47-66.

Garcés, Ma Pilar, 2006: "Las operacines de reformulación" en Milka Villayandre Llamazares (ed.): Actas del XXXV Simposio Internacional de la Sociedad Española de Lingüística, León: Universidad de León, 654-672.

Garcés, Ma Pilar, 2008: La organización del discurso: marcadores de ordenación y de reformulación, Madrid / Frankfurt: Iberoamericana / Vervuert.

Goldberg, Adele, 1995: Constructions. A Construction Grammar Approach to Argument Structure, Chicago/ London: The University of Chicago Press.

Gülich, Elisabeth y Thomas Kotschl, 1983: "Les marqueurs de reformulation paraphrastique”, Cahiers de Linguistique Française 5, 305-351.

Gülich, Elisabeth y Thomas KotsCHI, 1995: "Discourse Production in Oral Communications" en Uta Quasthoff (ed.): Aspects of Oral Communication, Berlin/Nueva York: Walter de Gruyter, 30-66. 
Kay, Paul y Charles Fillmore, 1999: "Grammatical Constructions and Linguistic Generalizations: The What's X Doing Y? Construction", Language 75, 1-33.

LeE, David, 2001: Cognitive Linguistics: An Introduction, Oxford: Oxford University Press.

LeVInson, Stephen, 2004 [2000]: Significados presumibles. La teoría de la implicatura conversacional generalizada, Madrid: Editorial Gredos.

LIneLL, Per, 2009: "Grammatical Constructions in Dialogue" en Alexander Bergs y Gabriele Diewald (eds.): Contexts and Constructions, AmsterdamPhiladelphia: John Benjamins, 97-110.

Monjour, Alf, 2011: "iAh, vaya! Ya llegamos a donde íbamos - Aha! Da sind wir beim springenden Punkt... ¡Vaya marcador del discurso!" en Heidi AsCHENBERg y Óscar LouReda (eds.): Marcadores del discurso: de la descripción a la definición, MadridFrankfurt: Iberoamericana/Vervuert, 343-373.

Ono, Tsuyoshi y Sandra Thompson, 1995: "What can conversation tell us about syntax?" en Phillip DAvis (ed.): Descriptive and theoretical modes in alternative linguistics, Amsterdam \& Philadelphia: John Benjamins, 213-271.

Polanco Martínez, Fernando, 2013a: "Redes polisémicas y niveles de interpretación: representación semántica de unidades lingüísticas complejas: el caso de vamos", ELUA 27, 199-250.

Polanco Martínez, Fernando, 2013b: "Vino mucha gente, buena bebida, buena música... vamos, que fue genial': La función de la partícula que en enunciados recapitulativos: el caso de <vamos/ vaya, que + enunciado verbal> en español coloquial", Onomázein 28, 128-142.

Polanco Martínez, Fernando, 2014: "Variación funcional y polisemia en la descripción de marcadores conversacionales en español peninsular: el caso de vaya", RSEL 44/2, 131-164.

Polanco Martínez, Fernando, en prensa: "Modalidad y refuerzo ilocutivo en los marcadores conversacionales 'vamos' y 'vaya'. La función de reafirmación proposicional", LEA 37/1, 5-29.

PoRTolés, José, 2001 [1998]: Marcadores del discurso, Barcelona: Ariel.

Portolés, José, 2004: Pragmática para hispanistas, Madrid: Síntesis.

Rossarl, Corinne, 1994: Les operations de reformulation: analysse du processus et des marques dans une perspective contrastive français-italien, Berna: Peter-lang.

Santos Rio, Luis, 2003: Diccionario de partículas, Salamanca: Luso Española de Ediciones.

Verschueren, Jef, 2000: "Notes on the role of metapragmatic awareness in language", Pragmatics 10: 4, 439-456.

Verschueren, Jef, 2003 [1999]: Para comprender la pragmática, Madrid: Gredos. 